\title{
COMPLEX $B$-SPLINE COLLOCATION METHOD FOR SOLVING WEAKLY SINGULAR VOLTERRA INTEGRAL EQUATIONS OF THE SECOND KIND
}

\author{
M. RAMEZANI, H. JAFARI, S.J. JOHNSTON, AND D. BALEANU \\ Received 18 December, 2014
}

\begin{abstract}
In this paper we propose a new collocation type method for solving Volterra integral equations of the second kind with weakly singular kernels. In this method we use the complex $B$ spline basics in collocation method for solving Volterra integral. We compare the results obtained by this method with exact solution. A few numerical examples are presented to demonstrate the effectiveness of the proposed method.
\end{abstract}

2010 Mathematics Subject Classification: 65R20; 45J05

Keywords: Volterra integral equation, complex $B$-spline, collocation method

\section{INTRODUCTION}

In this paper we consider the Volterra integral equation with the second kind weakly singular kernel, namely

$$
u(t)=f(t)+\int_{0}^{t} k(t, s) u(s) d s, \quad t \in(a, b],
$$

where $k(t, s)$ and $f(t)$ are known and $u(t)$ is unknown. The function $k(t, s)$ is called a polar kernel if

$$
k(t, s)=\frac{g(t, s)}{(t-s)^{\alpha}}, \quad \alpha \in(0,1),
$$

where $g$ is bounded on $s, g(t, t) \neq 0$ and for all

$$
t, s \in C[a, b] ; g(t, s) \in C([a, b] \times[a, b]) .
$$

We rewrite the equation (1.1) in the following operator form:

$$
(I-K) u=f
$$

where the operator $K$ is assumed to be compact on a Banach Space $X$ to $X$.

During the past few decades, this equation has been used to study various problems of mathematical chemistry and physics, such as reactions including stereology, heat conduction with mixed boundary conditions [10], crystal growth, electrochemistry, 
super fluidity and the radiation of heat [7], electrochemistry, semi-conductors, scattering theory, seismology, heat conduction, metallurgy, fluid flow, chemical reactions and population dynamics $[12,13,27]$, the particle transport problems of astrophysics, potential theory and Dirichlet problem, electrostatic and radiative heat transfer problems and in some engineering fields [29,30], astronomy, optics, computational electromagnetic, quantum mechanics, seismology image processing [1,6].

In addition a method with exponential order convergence rate has been developed by Riley [23] for Volterra integral equations of the form

$$
u(t)-\int_{a}^{t}(t-s)^{p-1} k(t, s) u(s) d s=f(t), \quad a \leq t \leq b,
$$

where the kernel is also assumed to be weakly singular and the solution $u$ is generally not differentiable at $t=a$. In [16], the equation (1.2) has been solved with fractional B-spline basics.

In most of the cases, it is difficult to obtain analytical solution of integral equations, therefore many numerical methods such as collocation method with different basics [2,19,20], orthogonal bases and wavelets [17,21], Galerkin methods have been developed to solve equation (1.1) [4-6, 14].

Recently, many different basic functions have been utilized to estimate the result of integral equations, such as modified quadrature [24], optimal homotopy asymptotic method [15], Tau approximate method [18].

Spline functions are very efficient and useful in signal processing, mathematical and computer graphics [8, 9, 22, 25, 26]. In [3], Blu and Unser gave an extension of $B$-splines to fractional orders and later in [11], Forster $e t$. al. gave an extension to complex power.

In this paper, we solve equation (1.1) by using complex $B$-spline to obtain approximate solution. The paper is organized as follows: In Section 2, we recall some basic definitions and theorems of complex $B$-splines and its properties. Section 3 is devoted to the solution of weakly singular integral equation of second kind using collocation methods with complex $B$-spline basics. In Section 4 , by considering numerical examples reported in our work, the accuracy of the proposed scheme is demonstrated.

\section{COMPLEX $B$-SPLINES}

In this section we state some definitions and theorems $[11,20]$ that will be used later in our work.

Definition 1. The inner product $\int \overline{f(x)} g(x) d x$ between two complex $L^{2}$ functions $f, g$ is denoted by $(f, g)$, and the associated Euclidean norm is written as $\|\cdot\|_{2}$.

Definition 2. The Riemann zeta function is defined as $\xi(s)=\sum_{n \geq 1} n^{-s}$ for all real $s>1$. 
Definition 3. The basic functions for Schoenberg's polynomial splines with uniform knots $[3,20]$ are defined as

$$
\beta^{n}(x)=\frac{1}{n !} \sum_{j=0}^{n+1}(-1)^{j}\left(\begin{array}{c}
n+1 \\
j
\end{array}\right)(x-j)_{+}^{n} \quad x \in \mathbb{R}, n \in \mathbb{N},
$$

where

$$
(x-j)_{+}^{n}=\left\{\begin{array}{lll}
(x-j)^{n} & \text { if } \quad x>j \\
0 & \text { if } \quad x \leq j .
\end{array}\right.
$$

Definition 4. $x_{+}^{z}$ denotes the truncated power function of complex degree $z$ with knot zero:

$$
x_{+}^{z}=\left\{\begin{array}{cc}
x^{\Re z} e^{i \Im z \ln x}, & x>0, \\
0, & \text { elsewhere }
\end{array}\right.
$$

Definition 5. The complex $B$-spline $\beta^{z}$ of complex degree $z$ is defined in $L^{2}(\mathbb{R})$ via its Plancherel transform as

$$
\hat{\beta}^{z}(\omega)=\left(\frac{1-e^{-i \omega}}{i \omega}\right)^{z+1},
$$

where $z=\alpha+i \gamma$ with parameters $\alpha, \gamma \in \mathbb{R}$ and $\alpha>\frac{-1}{2}$.

Theorem 1 (cf. [11]). The complex B-spline $\beta^{z}$ is well-defined, uniformly continuous and belongs to the space $L^{2}(\mathbb{R})$.

Theorem 2 (cf. [11]). The time domain representation of the complex $B$-spline $\beta^{z}$ is given by

$$
\beta^{z}(x)=\frac{1}{\Gamma(z+1)} \sum_{k \geq 0}(-1)^{k}\left(\begin{array}{c}
z+1 \\
k
\end{array}\right)(x-k)_{+}^{z} .
$$

This equation is valid pointwise for all $x \in \mathbb{R}$ and $L^{2}(\mathbb{R})$.

The complex $B$-splines generate dyadic multiresolution analysis; i.e. they generate a sequence of spaces:

$$
\{0\} \subset \ldots \subset V_{-1} \subset V_{0} \subset V_{1} \subset \ldots \subset L^{2}(\mathbb{R})
$$

with the following properties:

(1) $\cap_{i} V_{i}=\{0\}$ and $\overline{\cup_{i} V_{i}}=L^{2}(\mathbb{R})$,

(2) $f(\bullet) \in V_{i}$ if and only if $f\left(2^{-i} \bullet\right) \in V_{0}$,

(3) $f(\bullet) \in V_{0}$ if and only if $f(\bullet-k) \in V_{0}$ for all $k \in \mathbb{Z}$,

(4) there exists a function $\varphi \in V_{0}$, called a scaling function, such that $\varphi(\bullet-k)_{k \in \mathbb{Z}}$ forms an orthonormal basis of $V_{0}$.

$V_{i}$ is the complex $B$-spline of order $z \in \mathbb{C}$ with knot points $k \cdot 2^{i}, k \in \mathbb{Z}$. 
Theorem 3 (cf. [11]). Let Re $z>0$. Then the spaces

$$
V_{i}=\overline{\operatorname{span}\left\{\beta z\left(\frac{x-2^{i} k}{2^{i}}\right)\right\}^{L^{2}(\mathbb{R})}} \quad, i \in \mathbb{Z}
$$

form a dyadic multiresolution analysis with scaling function $\beta^{z}$.

The complex $B$-spline spaces at scale $a$ is defined as

$$
S_{a}^{z}=\left\{s_{a}: \exists c \in \ell^{2}, s_{a}(x)=\sum_{k \in \mathbb{Z}} c(k) \beta^{z}\left(\frac{x}{a}-k\right)\right\} .
$$

Then given an arbitrary function $f \in L^{2}(\mathbb{R})$, we determine its least-squares approximation in $S_{a}^{z}$ by applying the following orthogonal projection operator

$$
P_{a} f=\sum_{k \in \mathbb{Z}}\left(f, \frac{1}{a} \tilde{\beta}^{z}\left(\frac{\bullet}{a}-k\right)\right) \beta^{z}\left(\frac{\bullet}{a}-k\right) .
$$

This defines a projector because the functions $\beta^{z}$ and $\tilde{\beta}^{z}$ are biorthonormal [11], where $\tilde{\beta}^{z} \in S_{a}^{z}$ is the dual $B$-spline whose Fourier transform is

$$
\hat{\tilde{\beta}}^{z}(\bullet)=\frac{\hat{\beta}^{z}(\bullet)}{\sqrt{\sum_{k \in \mathbb{Z}}\left|\hat{\beta}^{z}(\bullet+2 \pi k)\right|^{2}}} .
$$

Theorem 4 (cf. [28]). The complex B-splines have a fractional order of approximation $\alpha+1$. Specifically, the least-squares approximation error is bounded by:

$$
\forall f \in W_{2}^{\alpha+1},\left\|f-P_{a} f\right\|_{2} \leq \frac{\sqrt{2 \xi(\alpha+2)-\frac{1}{2}}}{\pi^{\alpha+1}}\left\|D^{\alpha+1}\right\|_{2} a^{\alpha+1} .
$$

\section{THE COMPLEX $B$-SPLINE COLLOCATION METHOD}

To solve approximately the integral equation equation (1.1), we assume that $K$ is compact on a Banach space $X$ to $X$. We choose a finite dimensional family of functions $\tilde{u}(x)$ which is close to the exact solution $u(x)$. In practice, we choose a sequence of dimensional subspaces $X_{n} \subset X, n \geq 1$, with $X_{n}$ having dimension $d_{n}$. Let $X_{n}$ have a basis $\left\{\varphi_{1}, \ldots, \varphi_{d}\right\}$ with $d \equiv d_{n}$ for notational simplicity. We seek $u_{n}(x) \in X_{n}$, which can be written as

$$
u_{n}(x)=\sum_{j=0}^{d} c_{j} \varphi_{j}(x), \quad x \in D .
$$

This is substituted into equation (1.1), and coefficients $\left\{c_{1}, \ldots, c_{d}\right\}$ are determined by forcing the equation to be exact in some sense. For later use, we introduce

$$
r_{n}(x)=u_{n}(x)-\int_{D} k(x, s) u_{n}(s) d s-f(x),
$$




$$
=\sum_{j=1}^{d} c_{j}\left\{\varphi_{j}(x)-\int_{D} k(x, s) \varphi_{j}(s) d s\right\}-f(x), \quad x \in D .
$$

We pick distinct node $x_{1}, \cdots, x_{d} \in D$, and require

$$
r_{n}\left(x_{i}\right)=0, \quad i=1, \cdots, d .
$$

This leads to determining $\left\{c_{1}, \ldots, c_{d}\right\}$ as the solution of the linear system

$$
\sum_{j=1}^{d} c_{j}\left\{u\left(x_{i}\right)-\int_{D} k\left(x_{i}, s\right) u(s) d s\right\}=f\left(x_{i}\right), \quad i=1, \cdots, d .
$$

In following we show that this method can be used to solve equation (1.1), In this regard we give the following Lemma 1 and Theorem 5.

Lemma 1 (cf. [2]). Let $X$ be a Banach space and $P_{n}$ be a family of bounded projections on $X$ with

$$
P_{n} u \longrightarrow u \text { as } n \longrightarrow \infty, u \in X
$$

and $K: X \longrightarrow$ Xbe compact. Then

$$
\left\|K-P_{n} K\right\| \longrightarrow 0 \text { as } n \longrightarrow \infty .
$$

Theorem 5 (cf. [11]). If $G \in \mathbb{R}$ be an integral equation with a weakly singular kernel then it is a compact operator on $C(G)$, where $C(G)$ is space of continuous real or complex valued functions on compact subsets $G \in \mathbb{R}$.

Theorem 6. Equation (1.1) can be solved with collocation method by using complex B-spline basis.

Proof. If we introduce equation (3.1) to projection operator $P_{n}$ that maps $X$ onto $X_{n}$, define $P_{n} u(x)$ to be that element of $X_{n}$ that interpolates $X$ at the nodes $\left\{x_{1}, \ldots, x_{d}\right\}$. This means writing

$$
P_{n} u(x)=\sum_{j=1}^{d} c_{j} \varphi_{j}(x)
$$

with the coefficients $\left\{c_{j}\right\}$ determined by solving the linear system

$$
\sum_{j=1}^{d} c_{j} \varphi_{j}\left(x_{i}\right)=u\left(x_{i}\right), \quad i=1, \cdots, d
$$

Then this linear system has a unique solution if

$$
\operatorname{det}\left[\varphi_{j}\left(x_{j}\right)\right] \neq 0 \text {. }
$$

From Theorem 1, complex B-spline basis belong to $L^{2}(\mathbb{R})$ and with the help Theorem 2 this method is convergent. Then in view of Lemma 1 and Theorem 5 we can use collocation method for these type of integral equations. 
Now we can use collocation methods for solving weakly singular integral equation of second kind with complex B-spline basis.

In equation (1.1), let $X=L^{2}(\mathbb{R})$ and $V_{n}=X_{n}$. Then if $u(x) \in L^{2}(\mathbb{R})$ and $u_{n}(x) \in$ $V_{n}$, where

$$
u_{n}(x)=\sum_{j \in \mathbb{Z}} c(j) \beta^{z}\left(2^{n} x-j\right), \quad j \in \mathbb{Z},
$$

with $0 \leq t \leq b$ and $n \in \mathbb{N}$ then we have

$$
u_{n}^{2^{n}}(x)=\sum_{j=1-2^{n}}^{b} c(j) \beta^{z}\left(2^{n} x-j\right), \quad b \in \mathbb{R},
$$

with nodes $x_{i}=\frac{b i}{2^{n}}$. Then

$$
r_{n}^{2^{n}}\left(x_{i}\right)=\sum_{j=1-2^{n}}^{b} c_{j}\left\{u\left(x_{i}\right)-\int_{D} k\left(x_{i}, s\right) u(s) d s\right\}-f\left(x_{i}\right)=0 \quad i=0, \cdots, b .
$$

We define the absolute error

$$
E_{n}^{2^{n}}(u(x))=\left\|u(x)-u_{n}^{2^{n}}(x)\right\|_{2}=\left(\int_{0}^{b}\left|u(x)-u_{n}^{2^{n}}(x)\right|^{2}\right)^{\frac{1}{2}},
$$

and note that when $n \rightarrow \infty$ and $d \rightarrow \infty$ then $u_{n}^{2^{n}}(x) \rightarrow u(x)$.

Using the Theorem 1 and Lemma 1, the relatively error is defined as

$$
e_{n}=\frac{\max _{0 \leq i \leq 2^{n}}\left|E_{n}^{2^{n}}\left(u\left(x_{i}\right)\right)\right|}{\max _{0 \leq x \leq b}|u(x)|} .
$$

\section{ILLUSTRATIVE EXAMPLES}

In order to show better the theoretical results of the previous sections, we now consider the numerical solution of the equation (1.1), with various choices of $f(x)$ for $x \in[0,1]=D$. By using equation (3.6), we obtain $\left\{c_{1}, \ldots, c_{d}\right\}$. Then in view of (3.7) and (3.8) at several points of interval $D$ we obtain the absolute and the relative errors.

Example 1. Let $b=1, g(t, s)=t s$ and $f(x)=x(1-x)+\frac{16}{105} x^{\frac{7}{2}}(7-6 x)$ with the exact solution $u(x)=x(1-x)$. Table 1 shows the absolute errors obtained by the knot points $x_{i}=\frac{i}{2^{n}} ; i=0, \ldots, 2^{n}$ with $z=0.5+i$.

In Figure 1, the horizontal axis represents the $n$ index's $V_{n}$ the vertical axis represents the relative error $e_{n}$ is intentional, as can be seen, by increasing the index of $n$, the relative error decreases.

From Table 1 we see that the maximum error occurs at point $x=1$. We now show the relative error for different interval of $z$ this point $(x=1)$ in Tables 3,4 and 5 . 
TABLE 1. Absolute Errors

\begin{tabular}{|l|ccccc|}
\hline$x$ & $E_{0}^{2^{0}}(u(x))$ & $E_{1}^{2^{1}}(u(x))$ & $E_{2}^{2^{2}}(u(x))$ & $E_{3}^{2^{3}}(u(x))$ & $E_{4}^{2^{4}}(u(x))$ \\
\hline 0 & 0 & 0 & 0 & 0 & 0 \\
0.25 & 0.180375 & 0.119014 & 0.0013166 & 0.0008720 & 0.0005532 \\
0.5 & 0.1676936 & 0.126606 & 0.019329 & 0.013126 & 0.00670011 \\
0.75 & 0.1676936 & 0.126606 & 0.019329 & 0.0131267 & 0.00670011 \\
1 & 0.0720055 & 0.0578148 & 0.0425467 & 0.0263254 & 0.0111375 \\
\hline
\end{tabular}

TABLE 2. Relatively Errors

\begin{tabular}{|l|ccccc|}
\hline$n$ & 0 & 1 & 2 & 3 & 4 \\
\hline$e_{n}$ & 0.837556 & 0.506424 & 0.1701868 & 0.10530168 & 0.04455 \\
\hline
\end{tabular}

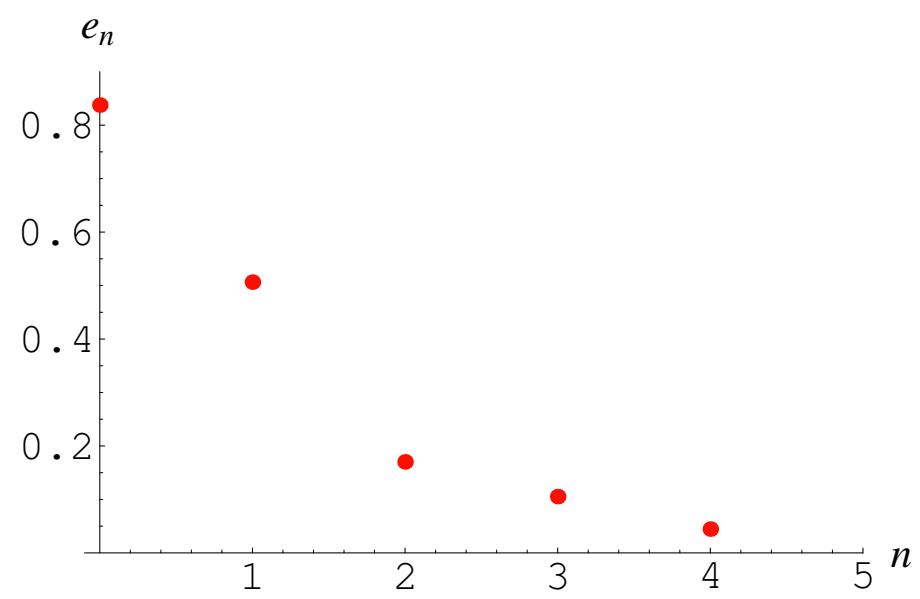

FIGURE 1. Points relative error in spaces $V_{n}$.

TABLE 3. The relative error for $|z|<1$

\begin{tabular}{|l|ccc|}
\hline$z$ & $0.1+0.01 \mathrm{i}$ & $0.5+0.1 \mathrm{i}$ & $0.2+0.9 \mathrm{i}$ \\
\hline$E_{4}^{2^{4}}(u(x))$ & 0.00913271 & 0.00301154 & 0.01920683 \\
\hline
\end{tabular}

TABLE 4. The relative error for $1 \leq|z|<2$

\begin{tabular}{|l|ccc|}
\hline$z$ & $1+\mathrm{i}$ & $1+0.5 \mathrm{i}$ & $1+0.9 \mathrm{i}$ \\
\hline$E_{4}^{2^{4}}(u(x))$ & 0.00745215 & 0.00248121 & 0.00842375 \\
\hline
\end{tabular}


TABLE 5. The relative error for $2 \leq|z|<3$

\begin{tabular}{|l|ccc|}
\hline$z$ & $2+0.1 \mathrm{i}$ & $2+0.5 \mathrm{i}$ & $2+1 \mathrm{i}$ \\
\hline$E_{4}^{2^{4}}(u(x))$ & 0.0054914 & 0.00949278 & 0.0301505 \\
\hline
\end{tabular}

We note that when $1 \leq|z|<2$, the error is minimum.

Example 2. Let $b=1, g(x, s)=1$ and $f(x)=\frac{1}{2} \pi x+\sqrt{x}$ with the exact solution $u(x)=\sqrt{x}$ and $z=0.5+0.5 i$. Table 6 shows the absolute error obtained by the knot points $x_{i}=\frac{i}{2^{n}}, \quad i=0, \ldots, 2^{n}$ with $z=0.5+0.5 i$.

TABLE 6. The absolute errors

\begin{tabular}{|l|ccccc|}
\hline $\mathrm{x}$ & $E_{0}^{2^{0}}(u(x))$ & $E_{1}^{2^{1}}(u(x))$ & $E_{2}^{2^{2}}(u(x))$ & $E_{3}^{2^{3}}(u(x))$ & $E_{4}^{2^{4}}(u(x))$ \\
\hline 0 & 0 & 0 & 0 & 0 & 0 \\
0.25 & 0.290563 & 0.126959 & 0.0413826 & 0.0199285 & 0.000803727 \\
0.5 & 0.168809 & 0.0702198 & 0.0333131 & 0.0130709 & 0.0044490 \\
0.75 & 0.0356813 & 0.0508203 & 0.00297352 & 0.00907127 & 0.00297352 \\
1 & 0.1156 & 0.0540076 & 0.0205252 & 0.0067599 & 0.00221725 \\
\hline
\end{tabular}

TABLE 7. The relatively error

\begin{tabular}{|l|ccccc|}
\hline$m$ & 0 & 1 & 2 & 3 & 4 \\
\hline$e_{m}$ & 0.290563 & 0.1269598 & 0.0413826 & 0.0199285 & 0.00803727 \\
\hline
\end{tabular}

In Figure 2, the horizontal axis represents the $n$ index's $V_{n}$ and vertical axis represents the relative error $e_{n}$ is intentional, as can be seen by increasing the index of $n$ relative error decreases.

From Table 6 we see that the maximum error occurs at point $x=0.5$. We now show the relative error for different interval of $z$ this point $(x=0.5)$ in Tables 8,9 and 10 .

TABLE 8. The relative error for $|z|<1$

\begin{tabular}{|lccc|}
\hline$z$ & $0.01+0.1 \mathrm{i}$ & $0.5+0.01 \mathrm{i}$ & $0.9+0.1 \mathrm{i}$ \\
\hline$E_{4}^{2^{4}}(u(x))$ & 0.0156468 & 0.000083763 & 0.00115488 \\
\hline
\end{tabular}

Here we note that when $|z|<1$, the error is minimum. 


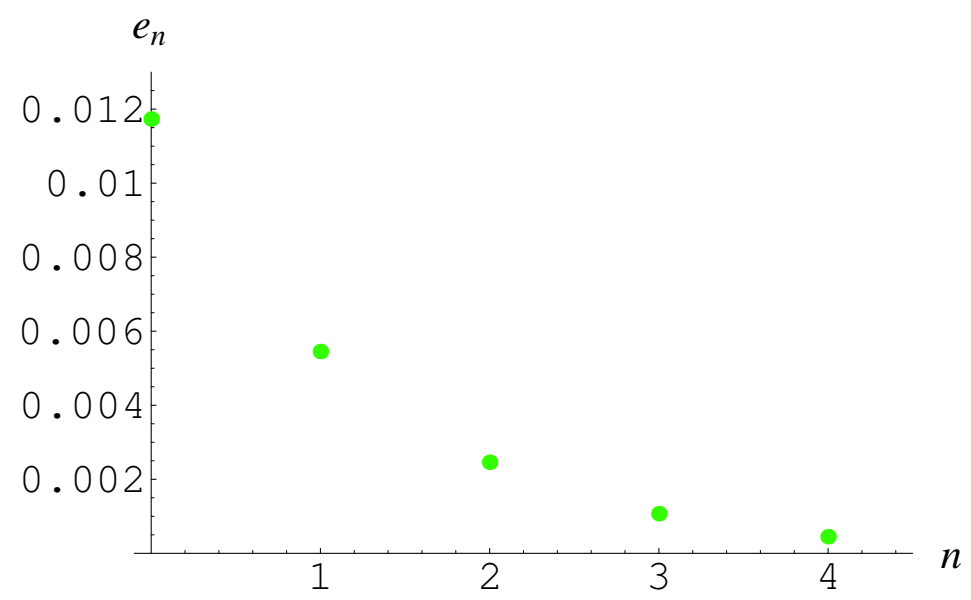

FIGURE 2. Points relative error in spaces $V_{n}$.

TABLE 9. The relative error for $1 \leq|z|<2$

\begin{tabular}{|l|ccc|}
\hline$z$ & $1+0.1 \mathrm{i}$ & $0.5+\mathrm{i}$ & $0.9+\mathrm{i}$ \\
\hline$E_{4}^{2^{4}}(u(x))$ & 0.00112826 & 0.019533 & 0.00526669 \\
\hline
\end{tabular}

TABLE 10. The relative error for $2 \leq|z|<3$

\begin{tabular}{|l|ccc|}
\hline$z$ & $1.5+\mathrm{i}$ & $2+0.1 \mathrm{i}$ & $2+0.5 \mathrm{i}$ \\
\hline$E_{4}^{2^{4}}(u(x))$ & 0.022596 & 0.0175306 & 0.0224039 \\
\hline
\end{tabular}

Example 3. Let $a=-1, b=1, g(x, s)=1$ and $f(x)=\sqrt{x+1}+\frac{1}{9}(x+1)^{\frac{3}{2}}\left(3 \ln (x+1)^{2}-\right.$ $16+\ln (4096))$ with the exact solution $u(x)=\sqrt{x+1}$.

Table 11 shows the absolute error obtained by the knot points $x_{i}=\frac{i}{2^{n}}-1, \quad i=$ $0, \ldots, 2^{n}$ with $z=1+0.5 i$.

TABLE 11. The absolute errors

\begin{tabular}{|l|ccccc|}
\hline$x$ & $E_{0}^{2^{0}}(u(x))$ & $E_{1}^{2^{1}}(u(x))$ & $E_{2}^{2^{2}}(u(x))$ & $E_{3}^{2^{3}}(u(x))$ & $E_{4}^{2^{4}}(u(x))$ \\
\hline-1 & 0 & 0 & 0 & 0 & 0 \\
\hline-0.5 & 0.016592 & 0.0056849 & 0.0026678 & 0.0012071 & 0.00052264 \\
0 & 0.016534 & 0.0077106 & 0.0034793 & 0.0015144 & 0.00063639 \\
0.5 & 0.007966 & 0.00063781 & 0.0030579 & 0.0013167 & 0.00060392 \\
1 & 0.019279 & 0.00068 & 0.0004755 & 0.00002275 & 0.00001357 \\
\hline
\end{tabular}


TABLE 12. The relative errors

\begin{tabular}{|l|ccccc|}
\hline$n$ & 0 & 1 & 2 & 3 & 4 \\
\hline$e_{n}$ & 0.0117325 & 0.0054522 & 0.0024602 & 0.0010708 & 0.00044999 \\
\hline
\end{tabular}

In Figure 3 , the horizontal axis represents the $n$ index's $V_{n}$, axis represents the relative error $e_{n}$ is intentional, as can be seen by increasing the index of $n$ relative error decreases.

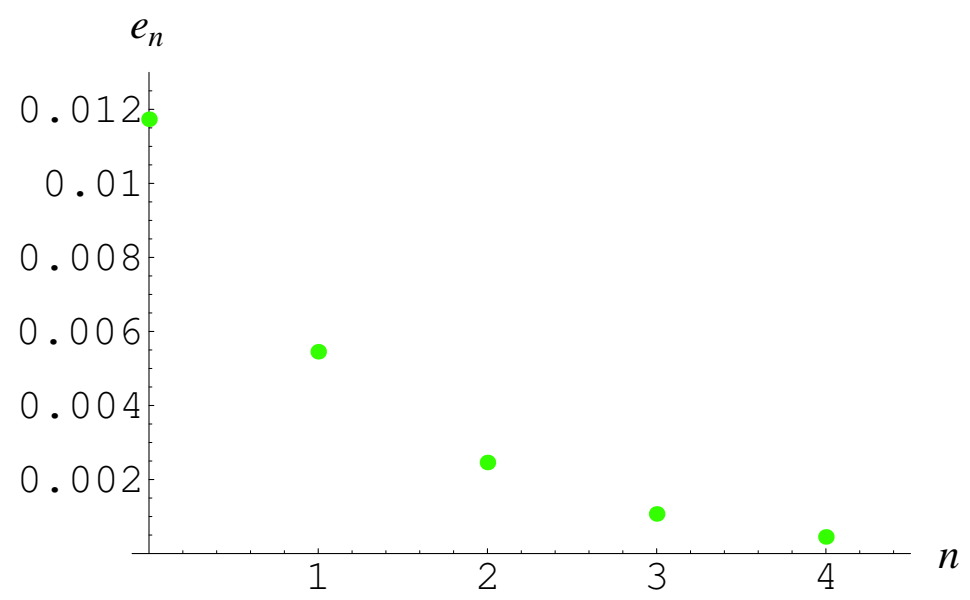

FIGURE 3. Points relative error in spaces $V_{n}$.

From Table 12 see that the maximum error occurs at point $x=0.5$. Now show the relative error for different interval of $z$ this point $(x=0.5)$ in Tables 13, 14 and 15 .

TABLE 13. The relative error for $|z|<1$

\begin{tabular}{|l|ccc|}
\hline$z$ & $0.1+0.1 \mathrm{i}$ & $0.5+0.1 \mathrm{i}$ & $0.2+0.9 \mathrm{i}$ \\
\hline$E_{4}^{2^{4}}(u(x))$ & 0.080492 & 0.00864855 & 0.086332 \\
\hline
\end{tabular}

TABLE 14. The relative error for $1 \leq|z|<2$

\begin{tabular}{|l|ccc|}
\hline$z$ & $0.1+\mathrm{i}$ & $1+0.5 \mathrm{i}$ & $0.9+\mathrm{i}$ \\
\hline$E_{4}^{2^{4}}(u(x))$ & 0.100855 & 0.0415961 & 0.0482324 \\
\hline
\end{tabular}

We see that when $|z|<1$, the error is minimum. 
TABLE 15 . The relative error for $2 \leq|z|<3$

\begin{tabular}{|l|ccc|}
\hline$z$ & $1+\mathrm{i}$ & $2+0.5 \mathrm{i}$ & $2+\mathrm{i}$ \\
\hline$E_{4}^{2^{4}}(u(x))$ & 0.0298086 & 0.0398539 & 0.0419062 \\
\hline
\end{tabular}

\section{CONCLUSION}

In this paper, we proposed an efficient algorithm for solving Volterra integral equations of second kind with weakly singular kernels by collocation type method. We used complex $B$-spline basics as basic functions in the collocation method. This approach gives better solution with respect to ordinary $B$-spline basics function. We presented three numerical examples which demonstrated That our proposal method is very attractive. Mathematica has been used in this paper for computation.

\section{REFERENCES}

[1] V. M. Aleksandrov and E. V. Kovalenko, "Mathematical method in the displacement problem," Inzh. Zh. Mekh. Tverd. Tela, vol. 2, pp. 77 - 89, 1984.

[2] K. Atkinson, The Numerical Solution of Integral Equations of the Second Kind, ser. Cambridge Monographs on Applied and Computational Mathematics. Cambridge University Press, 1997. doi: 10.1017/CBO9780511626340.

[3] T. Blu and M. Unser, "Quantitative Fourier analysis of approximation techniques: Part I - Interpolators and projectors,” IEEE Transactions on Signal Processing, vol. 47, no. 10, pp. 2783-2795, October 1999.

[4] H. Brunner, "The numerical solution of weakly singular Volterra integral equations by collocation on graded meshes," Mathematics of Computation, vol. 45, no. 172, pp. 417-437, 1985, doi: 10.1090/S0025-5718-1985-0804933-3.

[5] H. Brunner, Collocation methods for Volterra integral and Related Functional Differential Equations, ser. Cambridge Monographs on Applied and Computational Mathematics. Cambridge University Press, 2004.

[6] H. Brunner, "On the numerical solution of first-kind Volterra integral equations with highly oscillatory kernels," Isaac Newton Institute, HOP, pp. 13-17, 2010.

[7] Z. Chen and W. Jiang, "Piecewise homotopy perturbation method for solving linear and nonlinear weakly singular VIE of second kind," Applied Mathematics and Computation, vol. 217, no. 19, pp. 7790 - 7798, 2011, doi: 10.1016/j.amc.2011.02.086.

[8] C. K. Chui, Multivariate Splines, ser. CBMS-NSF Regional Conference Series in Applied Mathematics. Society for Industrial and Applied Mathematics, 1988, vol. 2, doi: 10.1137/1.9781611970173.

[9] C. de Boor, K. Höllig, and S. Riemenschneider, Box Splines, ser. Applied Mathematical Sciences. Springer, 1993, vol. 98.

[10] T. Diogo, N. B. Franco, and P. Lima, "High order product integration methods for a Volterra integral equation with logarithmic singular kernel," Communications on Pure and Applied Analysis, vol. 3, no. 2, pp. 217-235, 2004, doi: 10.3934/cpaa.2004.3.217.

[11] B. Forster, T. Blu, and M. Unser, "Complex B-splines," Applied and Computational Harmonic Analysis, vol. 20, no. 2, pp. 261 - 282, 2006, Computational Harmonic Analysis - Part 3, doi: 10.1016/j.acha.2005.07.003. 
[12] R. Gorenflo, Computation of rough solutions of Abel integral equations, ser. Preprint / A: Mathematik. Freie Univ., Fachbereich Mathematik, 1986, vol. 235.

[13] R. Gorenflo and S. Vessella, Abel Integral Equations, ser. Lecture Notes in Mathematics. Springer-Verlag, 1991. doi: 10.1007/BFb0084665.

[14] I. G. Graham, "Galerkin methods for second kind integral equations with singularities," Mathematics of Computation, vol. 39, no. 160, pp. 519-533, 1982, doi: 10.1090/S0025-5718-19820669644-3.

[15] M. S. Hashmi, N. Khan, and S. Iqbal, "Numerical solutions of weakly singular Volterra integral equations using the optimal homotopy asymptotic method," Computers \& Mathematics with Applications, vol. 64, no. 6, pp. 1567 - 1574, 2012, doi: 10.1016/j.camwa.2011.12.084.

[16] H. Jafari, C. M. Khalique, M. Ramezani, and H. Tajadodi, "Numerical solution of fractional differential equations by using fractional B-spline," Central European Journal of Physics, vol. 11, no. 10, pp. 1372-1376, 2013, doi: 10.2478/s11534-013-0222-4.

[17] Z. H. Jiang and W. Schaufelberger, Block pulse functions and their applications in control systems, ser. Lecture Notes in Control and Information Sciences. Springer-Verlag, 1992, vol. 179, doi: 10.1007/BFb0009162.

[18] S. Karimi Vanani and F. Soleymani, "Tau approximate solution of weakly singular Volterra integral equations," Mathematical and Computer Modelling, vol. 57, no. 3-4, pp. 494 - 502, 2013, doi: 10.1016/j.mcm.2012.07.004.

[19] R. Kress, Linear Integral Equations, ser. Applied Mathematical Sciences. Springer, 1999, vol. 82.

[20] P. Kythe and P. Puri, Computational Methods for Linear Integral Equations. Birkhäuser, 2002. doi: 10.1007/978-1-4612-0101-4.

[21] K. Maleknejad and M. Hadizadeh, "A new computational method for Volterra-Fredholm integral equations," Computers \& Mathematics with Applications, vol. 37, no. 9, pp. 1 - 8, 1999, doi: 10.1016/S0898-1221(99)00107-8.

[22] G. Nürnberger, Approximation by spline functions. Springer-Verlag, 1989. doi: 10.1002/zamm.19920720227.

[23] B. V. Riley, "The numerical solution of Volterra integral equations with nonsmooth solutions based on sinc approximation," Applied Numerical Mathematics, vol. 9, no. 3-5, pp. 249 - 257, 1992, doi: 10.1016/0168-9274(92)90019-A.

[24] J. Saberi Najafi and M. Heidari, "A new modied quadrature method for solving linear weakly singular integral equations," World Journal of Modeling and Simulaton, vol. 10, no. 1, pp. 69-78, February 2014.

[25] W. Schempp, Complex contour integral representation of cardinal spline functions, ser. Contemporary Mathematics. American Mathematical Society, 1982.

[26] I. Schoenberg, "Contributions to the problem of approximation of equidistant data by analytic functions," in I. J. Schoenberg Selected Papers, ser. Contemporary Mathematicians, C. de Boor, Ed. Birkhäuser Boston, 1988, pp. 3-57, doi: 10.1007/978-1-4899-0433-1.

[27] H. J. J. Te Riele, "Collocation methods for weakly singular second-kind Volterra integral equations with non-smooth solution," IMA Journal of Numerical Analysis, vol. 2, no. 3, pp. 437-449, 1982, doi: 10.1093/imanum/2.4.437.

[28] M. Unser and T. Blu, "Fractional splines and wavelets," SIAM Rev., vol. 42, no. 1, pp. 43-67, 2000, doi: $10.1137 /$ S0036144598349435.

[29] W. Wang, "Mechanical algorithm for solving the second kind of Volterra integral equation," Applied Mathematics and Computation, vol. 173, no. 2, pp. 1149 - 1162, 2006, doi: 10.1016/j.amc.2005.04.060.

[30] A. M. Wazwaz and S. A. Khuri, "A reliable technique for solving the weakly singular second-kind Volterra-type integral equations," Applied Mathematics and Computation, vol. 80, no. 2-3, pp. 287 - 299, 1996, doi: 10.1016/0096-3003(95)00279-0. 
Authors' addresses

\section{Ramezani}

M. Ramezani, Young Researchers and Elite Club, Parand Branch, Islamic Azad University, Tehran, Iran

E-mail address: mr_63_900yahoo.com

\section{H. Jafari}

Department of Mathematics, University of Mazandaran, Babolsar, Iran

Current address: Department of Mathematical Sciences, University of South Africa, PO Box 392,

UNISA 0003, Johannesburg, South Africa

E-mail address: jafarh@unisa.ac.za

\section{S.J. Johnston}

S.J. Johnston, Department of Mathematical Sciences, University of South Africa, PO Box 392, UNISA 0003, Johannesburg, South Africa

E-mail address: johnss jeunisa.ac.za

\section{Baleanu}

D. Baleanu, Department of Mathematics and Computer Science, Çankaya University, Ankara, Turkey

Current address: Institute of Space Sciences, PO Box MG-23, R 76900, Magurele-Bucharest, Romania

E-mail address: dumitru@cankaya.edu.tr 